\title{
A NONSTANDARD RESONANCE PROBLEM FOR ORDINARY DIFFERENTIAL EQUATIONS
}

\author{
SHAIR AHMAD
}

\begin{abstract}
Necessary and sufficient conditions are established for the existence of bounded solutions for a class of second order differential equations.
\end{abstract}

\section{INTRODUCTION AND SUMMARY}

This paper is a study of a Landesman-Lazer type problem for an ordinary differential equation in which the usual methods of nonlinear analysis such as fixed point theory, degree theory, monotone operator theory, and critical point theory do not seem to be applicable.

We consider the differential equation

$$
y^{\prime \prime}(t)+c y^{\prime}(t)+g(y(t))=p(t)
$$

where $p$ and $g$ are continuous functions defined on $(-\infty, \infty)$ satisfying certain conditions given below, and $c$ is a constant. We shall study the problem of the existence of solutions of (1.1) that are bounded on a ray $t_{0} \leq t<\infty$ and those that are bounded on $(-\infty, \infty)$.

In order to motivate our main results, we first recall some known results for the case where $p$ is periodic. Suppose that the period of $p$ is $L, L>0$, and let

$$
p_{0}=\frac{1}{L} \int_{0}^{L} p(t) d t .
$$

It follows from a result of Lazer [6] that if

$$
g(\xi) / \xi \rightarrow 0 \text { as }|\xi| \rightarrow \infty,
$$

and there exists $\xi_{1}, \xi_{1} \geq 0$, such that

$$
\left(g(\xi)-p_{0}\right) \xi \geq 0 \text { if }|\xi| \geq \xi_{1}
$$

then for any value of $c$-even for $c=0$-there exists at least one $L$-periodic solution of (1.1). Independently, Bebernes and Martelli [1] and Ward [11] established results which show that if $c \neq 0$, then condition (1.3) alone guarantees the existence of a $T$-periodic solution of (1.1). This was also contained in the

Received by the editors February 14, 1989.

1980 Mathematics Subject Classification (1985 Revision). Primary 34A34; Secondary 34C11. 
work of N. Rouche and J. Mawhin [8], where they studied a more general system of differential equations. The proofs of all of the above-cited results use some method of compactness.

In this paper we shall consider nonlinearities $g$ of the type where the limits

$$
\lim _{\xi \rightarrow \infty} g(\xi)=g(\infty), \quad \lim _{\xi \rightarrow-\infty} g(\xi)=g(-\infty)
$$

exist in the broad sense that they are possibly infinite and that for $\xi \in(-\infty, \infty)$

$$
g(-\infty) \leq g(\xi) \leq g(\infty) .
$$

In engineering literature such functions model saturation (see $\S 6$ ).

If $p(t)$ is $L$-periodic and $p_{0}$ is defined as above, it follows that the condition

$$
g(-\infty)<p_{0}<g(\infty),
$$

usually referred to as a Landesman-Lazer condition, implies (1.3), and is therefore sufficient for the existence of an $L$-periodic solution of (1.1) if $c \neq 0$. Conversely, if one assumes that (1.1) has an $L$-periodic solution $y_{0}(t)$, it follows from integrating the differential equation from 0 to $L$ that

$$
\frac{1}{L} \int_{0}^{L} g\left(y_{0}(t)\right) d t=p_{0} .
$$

Therefore, assuming (1.4), this implies that the condition

$$
g(-\infty) \leq p_{0} \leq g(\infty)
$$

is necessary for the existence of an $L$-periodic solution of (1.1). If (1.4) is replaced by the stronger condition that for all $\xi, \xi \in(-\infty, \infty)$,

$$
g(-\infty)<g(\xi)<g(\infty)
$$

then (1.6) implies that (1.5) holds. Therefore, if $(1.4)^{\prime}$ holds and $c \neq 0$, then (1.5) is both necessary and sufficient for the existence of an $L$-periodic solution.

In this paper we always assume that either (1.4) or $(1.4)^{\prime}$ holds and that $c>0$. Results for which $c<0$ and for which the inequalities in (1.4) and $(1.4)^{\prime}$ are reversed can also be obtained, but these conditions do not seem to have physical meaning. We replace the condition that $p(t)$ be periodic by the assumption that $p(t)$ be continuous and bounded on $(-\infty, \infty)$ and that there exist a number $p_{0}$ such that for any $a \in(-\infty, \infty)$

$$
\lim _{T \rightarrow \infty} \int_{a}^{a+T} p(t) d t=p_{0}
$$

uniformly with respect to $a$. If $p(t)$ happens to be $L$-periodic, then this condition holds and $p_{0}$ is the same number as defined earlier. More generally, condition (1.8) will hold if $p(t)$ is almost periodic (see [2]), but this is not necessary. For example, (1.8) will hold if $p(t)$ is the sum of an almost periodic function and a function in $L^{1}(-\infty, \infty)$. Our main result shows that given $t_{0} \in(-\infty, \infty)$, if (1.4) holds then the strict inequalities (1.5) are sufficient and 
the weak inequalities are necessary for the existence of a solution bounded on $\left[t_{0}, \infty\right)$. Moreover, if $(1.4)^{\prime}$ holds then the inequalities (1.5) are both necessary and sufficient for the existence of a solution bounded on $\left[t_{0}, \infty\right)$.

Unlike any of the above-cited references, we show that conditions (1.5) have an implication for the totality of solutions of $(1.1)$ defined on $\left[t_{0}, \infty\right)$ : namely, they are all bounded on this interval. Also, we show that (1.5) implies the existence of a solution of (1.1) which is bounded on $(-\infty, \infty)$.

Example. From the above-quoted theorems one might conjecture that if $p(t)$ satisfies the above conditions and there exists $\xi_{1} \geq 0$ such that (1.3) holds, then there exists a solution of $(1.1)$ bounded on $(-\infty, \infty)$. However, this is false for the following reason: There exists an almost periodic function $p(t)$ such that $p_{0}=0$, but the integral $\int_{0}^{t} p(s) d s$ is unbounded on $(-\infty, \infty)$ (see $[3, \mathbf{p}$. 72]). If $g(\xi) \equiv 0$, then (1.4) holds and hence, by what is shown in the next section, for any solution $y(t)$ of $y^{\prime \prime}(t)+c y^{\prime}(t)=p(t), y^{\prime}(t)$ is bounded on $[0, \infty)$ provided $c>0$. Since

$$
y^{\prime}(t)+c y(t)=y^{\prime}(0)+c y(0)+\int_{0}^{t} p(s) d s
$$

it follows that $y(t)$ cannot be bounded on $[0, \infty)$.

As a byproduct of some of the estimates needed to prove our main theorem we show that if $g$ is an arbitrary continuous function and there exists an isolated zero $\xi_{0}$ of the equation $g(\xi)-p_{0}=0$ such that $\left(g(\xi)-p_{0}\right)\left(\xi-\xi_{0}\right)>0$ for $\xi \neq \xi_{0}$ and $\left|\xi-\xi_{0}\right|$ small, then for any $\alpha>0$ there exist numbers $\gamma_{1}>0$, $\gamma_{2}>0$ and $c^{*}>0$ such that if $t_{0} \in(-\infty, \infty), c \geq c^{*}$, and $y(t)$ is a solution of (1.1) satisfying the inequalities $\left|y\left(t_{0}\right)-p_{0}\right|<\gamma_{1}$ and $\left|y^{\prime}\left(t_{0}\right)\right|<\gamma_{2}$, then $\left|y(t)-p_{0}\right|<\alpha$ and $\left|y^{\prime}(t)\right|<\alpha$ for $t \geq t_{0}$.

It follows from a theorem due to Reuter [9] that if $g(-\infty)=-\infty, g(\infty)=$ $\infty$ and $p$ is only assumed to be continuous and bounded on $\left[t_{0}, \infty\right)$, then all solutions of $(1.1)$ are bounded on $\left[t_{0}, \infty\right)$. However, we include the case $g( \pm \infty)= \pm \infty$ in our discussion for the sake of completeness.

Another nonstandard resonance problem was considered by Frederickson and Lazer in [4], in which it was assumed that the restoring force be linear and the damping nonlinear. They showed that if $f \geq 0, f \not \equiv 0, f$ is continuous on $(-\infty, \infty)$ and $p(t)$ is almost periodic, then a necessary and sufficient condition that

$$
y^{\prime \prime}(t)+f(y(t)) y^{\prime}(t)+y(t)=p(t)
$$

have an almost periodic solution is that

$$
F(\infty)-F(-\infty)>2 \sqrt{a_{1}^{2}+b_{1}^{2}}
$$

where $F(0)=0, F^{\prime}(\xi)=f(\xi)$, and

$$
\left\{\begin{array}{l}
a_{1} \\
b_{1}
\end{array}=\lim _{T \rightarrow \infty} \frac{1}{T} \int_{0}^{T} p(t)\left\{\begin{array}{l}
\cos t \\
\sin t
\end{array} d t .\right.\right.
$$


Although our methods and those of [4] both involve a basic theory of analysis and differential equation, the proofs as well as the equations considered are fundamentally different.

We emphasize that since $g$ is only assumed to be continuous, the solutions of the initial value problem associated with (1.1) are, in general, not unique. However, this does not introduce any technical complications.

\section{BOUNDEDNESS OF THE DERIVATIVE}

In this section we assume that $p$ and $g$ are defined and continuous on $(-\infty, \infty)$, that $g$ has limits $g( \pm \infty)$, which are possibly infinite, that $p$ is bounded, and

$$
g(-\infty) \leq g(\xi) \leq g(\infty), \quad-\infty<\xi<\infty .
$$

Let $c_{1}>0$ be a constant such that

$$
|p(t)| \leq c_{1}, \quad t \in(-\infty, \infty) .
$$

We consider the differential equation

$$
y^{\prime \prime}(t)+c y^{\prime}(t)+g(y(t))=p(t)
$$

where it is always assumed that $c>0$. If $t_{0} \in(-\infty, \infty)$ and $y_{0}$ and $v_{0}$ are given numbers, then by a solution of the initial value problem for (2.3) determined by the initial conditions

$$
y\left(t_{0}\right)=y_{0}, \quad y^{\prime}\left(t_{0}\right)=v_{0}
$$

we mean a solution whose interval of existence is maximal.

Theorem 2.1. For arbitrary $t_{0}, y_{0}$, and $v_{0}$, the interval of existence of the initial value problem given by (2.3) and (2.4) contains $\left[t_{0}, \infty\right)$. There exists $k>0$ such that for any $A>0$ and $B>0$ there exists $T(A, B)>0$ such that if $t_{0} \in(-\infty, \infty)$ and $y(t)$ is a solution of the initial value problem (2.3)-(2.4) and

$$
\left|y_{0}\right| \leq A, \quad\left|v_{0}\right| \leq B
$$

then

$$
t \geq t_{0}+T(A, B) \Rightarrow\left|y^{\prime}(t)\right| \leq k .
$$

Given $A>0$ and $B>0$, there exists $b(A, B)>0$ such that if $t_{0} \in(-\infty, \infty)$ and $y(t)$ is a solution of problem (2.3)-(2.4) and conditions (2.5) hold, then

$$
\left|y^{\prime}(t)\right| \leq b(A, B), \quad t \geq t_{0} .
$$

Proof. We defer the part of the proof concerning the interval of existence of solutions of (2.3)-(2.4) to the end. Until then we assume that $y$ is a solution of $(2.3)$ which exists on $\left[t_{0}, \infty\right)$.

There are four cases to consider.

Case I. Suppose that

$$
-\infty<g(-\infty) \leq g(\infty)<\infty .
$$


If $c_{2}, c_{2}>0$, is a constant such that $g(\xi) \leq c_{2}$ for all $\xi \in(-\infty, \infty)$, and $c_{3}=c_{1}+c_{2}$, then it follows from (2.2) and (2.3) that $-c_{3} \leq y^{\prime \prime}(t)+c y^{\prime}(t) \leq c_{3}$ for all $t \geq t_{0}$. Multiplying these inequalities by $e^{c t}$, integrating from $t_{0}$ to $t$, and making some obvious manipulations, we obtain the inequalities

$$
\left(-c_{3} / c\right)\left(1-e^{-c\left(t-t_{0}\right)}\right) \leq y^{\prime}(t)-y^{\prime}\left(t_{0}\right) e^{-c\left(t-t_{0}\right)} \leq\left(c_{3} / c\right)\left(1-e^{-c\left(t-t_{0}\right)}\right)
$$

from which it follows that if $\left|y^{\prime}\left(t_{0}\right)\right| \leq B$, then

$$
\left|y^{\prime}(t)\right| \leq \max \left\{B, c_{3} / c\right\} \equiv b(B), \quad t \geq t_{0},
$$

which proves (2.7). The same inequalities imply that for any $B>0$, there exists a number $T=T(B)$ such that if $\left|y^{\prime}\left(t_{0}\right)\right| \leq B$, then

$$
t \geq t_{0}+T(B) \Rightarrow\left|y^{\prime}(t)\right| \leq 2 c_{3} / c
$$

which is (2.6) with $k=2 c_{3} / c$.

Case II. Suppose that

$$
-\infty<g(-\infty)<g(\infty)=\infty .
$$

Then there exists a number $c_{3}>0$ such that $p(t)-g(\xi) \leq c_{3}$ for all $t \in$ $(-\infty, \infty)$ and all $\xi \in(-\infty, \infty)$. Consequently, $y^{\prime \prime}(t)+c y^{\prime}(t) \leq c_{3}$ for all $t \geq t_{0}$. An argument similar to that in Case I shows that

$$
y^{\prime}(t) \leq y^{\prime}\left(t_{0}\right) e^{-c\left(t-t_{0}\right)}+\left(c_{3} / c\right)\left(1-e^{-c\left(t-t_{0}\right)}\right), \quad t \geq t_{0} .
$$

As in Case I, this implies that if $y^{\prime}\left(t_{0}\right) \leq B$, then there exists a number $T_{1}=$ $T_{1}(B)$ such that

$$
t \geq t_{0}+T_{1}(B) \Rightarrow y^{\prime}(t) \leq 2 c_{3} / c
$$

and

$$
y^{\prime}(t) \leq \max \left\{B, c_{3} / c\right\} \equiv d_{1}(B), \quad t \geq t_{0} .
$$

To prove the assertions of the theorem in Case II, we make use of some lemmas.

Let $\xi_{0}$ be chosen so large that

$$
\xi \geq \xi_{0} \Rightarrow g(\xi) \geq c_{1}+1 .
$$

Lemma 2.1. If $y\left(t_{0}\right) \leq A$ and $y^{\prime}\left(t_{0}\right) \leq B$, then

$$
y(t) \leq \max \left\{A, \xi_{0}\right\}+\frac{1}{c} d_{1}(B) \equiv d_{2}(A, B), \quad t \geq t_{0} .
$$

Proof. Assuming the contrary, there exist numbers $t_{1}$ and $t_{2}$ with $t_{0} \leq t_{1}<t_{2}$ such that $y\left(t_{1}\right)=\max \left\{A, \xi_{0}\right\}, y\left(t_{2}\right)=y\left(t_{1}\right)+\frac{1}{c} d_{1}(B)$, and $y\left(t_{1}\right)<y(t)<y\left(t_{2}\right)$ for $t_{1} \leq t \leq t_{2}$. Since $y^{\prime \prime}(t)+c y^{\prime}(t)=p(t)-g(y(t)) \leq-1$ for $t_{1} \leq t \leq t_{2}$, we have

$$
y^{\prime}\left(t_{2}\right)-y^{\prime}\left(t_{1}\right)+c\left[y\left(t_{2}\right)-y\left(t_{1}\right)\right]<0 .
$$


Clearly, $y^{\prime}\left(t_{2}\right) \geq 0$, and hence from (2.12) and (2.15) we obtain $y\left(t_{2}\right)<y\left(t_{1}\right)+$ $\frac{1}{c} y^{\prime}\left(t_{1}\right) \leq y\left(t_{1}\right)+\frac{1}{c} d_{1}(B)$, which is a contradiction. This proves the lemma.

According to $(2.8)^{2}, c_{4}=c_{4}(A, B)>0$ can be chosen so that if $-\infty<\xi \leq$ $d_{2}(A, B)$, then $|g(\xi)| \leq c_{4}(A, B)$. Therefore, if $c_{5}=c_{5}(A, B)=c_{4}(A, B)+c_{1}$, then it follows from Lemma 2.1 that $y\left(t_{0}\right) \leq A$ and $y^{\prime}\left(t_{0}\right) \leq B$ imply that $-c_{5} \leq y^{\prime \prime}(t)+c y^{\prime}(t) \leq c_{5}$ for all $t \geq t_{0}$. As in Case I, it follows that if $y\left(t_{0}\right) \leq A$ and $\left|y^{\prime}\left(t_{0}\right)\right| \leq B$, then

$$
\left|y^{\prime}(t)\right| \leq \max \left\{B, c_{5}(A, B) / c\right\}=b(A, B), \quad t \geq t_{0} .
$$

This establishes (2.7) in Case II.

In order to establish (2.6), we use

Lemma 2.2. If $y\left(t_{0}\right) \leq A, y^{\prime}\left(t_{0}\right) \leq B$, and

$$
L=L(A, B)=1+(c+1)\left(d_{2}(A, B)-\xi_{0}\right)+d_{1}(B),
$$

then every subinterval of $\left[t_{0}, \infty\right)$ of length $L$ contains a point $t^{*}$ such that $y\left(t^{*}\right) \leq \xi_{0}$.

Proof. Suppose that $t_{1} \in\left[t_{0}, \infty\right)$ and, contrary to the assumption of the lemma, $y(t)>\xi_{0}$ for all $t$ in $\left[t_{1}, t_{1}+L\right]$. For $t$ in this range we have $y^{\prime \prime}(t)+c y^{\prime}(t)=$ $p(t)-g(y(t)) \leq-1$, and hence, by integration,

$$
y^{\prime}(t) \leq y^{\prime}\left(t_{1}\right)+c\left(y\left(t_{1}\right)-y(t)\right)-\left(t-t_{1}\right) .
$$

From (2.12) and (2.14) we have

$$
y^{\prime}(t) \leq d_{1}(B)+c\left(d_{2}(A, B)-\xi_{0}\right)-\left(t-t_{1}\right)
$$

for $t \in\left[t_{1}, t_{1}+L\right]$. Setting $\bar{t}=t_{1}+d_{1}(B)+c\left(d_{2}(A, B)-\xi_{0}\right)+1$, it follows that for $\bar{t} \leq t \leq t_{1}+L$ we have $y^{\prime}(t) \leq-1$. Hence,

$$
y\left(t_{1}+L\right) \leq y(\bar{t})-\left(t_{1}+L-\bar{t}\right) \leq d_{2}(A, B)-\left(t_{1}+L-\bar{t}\right)=\xi_{0},
$$

which is a contradiction. This completes the proof of the lemma.

Let $A>0, B>0$, and assume that $y\left(t_{0}\right) \leq A$, and $y^{\prime}\left(t_{0}\right) \leq B$. From (2.11) we have $y^{\prime}(t) \leq 2 c_{3} / c$ for $t \geq t_{0}+T_{1}(B)$, and, by what we just established above, there exists a number $t^{*}, t^{*} \in\left[t_{0}+T_{1}(B), t_{0}+T_{1}(B)+L(A, B)\right]$ such that $y\left(t^{*}\right) \leq \xi_{0}$. Applying Lemma 2.1, with $t_{0}$ replaced by $t^{*}$, it follows that

$$
y(t) \leq \xi_{0}+\frac{1}{c} d_{1}\left(\frac{2 c_{3}}{c}\right)=\xi_{0}+\frac{2 c_{3}}{c^{2}}
$$

for all $t \geq t^{*}$. Consequently, if

$$
c_{6}=\sup \left\{g(\xi) \mid-\infty<\xi<\xi_{0}+2 c_{3} / c^{2}\right\}<\infty
$$

and $c_{7}=c_{1}+c_{6}$, then $-c_{7} \leq y^{\prime \prime}(t)+c y(t)<c_{7}$ for $t \geq t^{*}$. Using an argument similar to that in Case I, we obtain the inequalities

$$
\left(-c_{7} / c\right)\left(1-e^{-c\left(t-t^{*}\right)}\right) \leq y^{\prime}(t)-y^{\prime}\left(t_{0}\right) e^{-c\left(t-t^{*}\right)} \leq\left(c_{7} / c\right)\left(1-e^{-c\left(t-t^{*}\right)}\right) .
$$


It is easy to see that for $t \geq t^{*}$ there exists a number $T_{2}=T_{2}(A, B)$ such that $\left|y^{\prime}(t)\right| \leq 2 c_{7} / c$ for all $t \geq t^{*}+T_{2}(A, B)$. Recalling that $t_{0}<t^{*}<t_{0}+T_{1}(B)+$ $L(A, B)$, it follows that if we set $T(A, B)=T_{1}(B)+T_{2}(A, B)+L(A, B)$, then

$$
t \geq t_{0}+T(A, B) \Rightarrow\left|y^{\prime}(t)\right| \leq 2 c_{7} / c .
$$

This proves (2.6) with $k=2 c_{7} / c$.

Case III. Suppose that

$$
-\infty=g(-\infty)<g(\xi) \leq g(\infty)<\infty, \quad \xi \in(-\infty, \infty) .
$$

If we set $\widehat{g}(\xi)=-g(-\xi)$, then for $\xi \in(-\infty, \infty)$ we have $-\infty<\widehat{g}(-\infty) \leq$ $g(\xi)<\widehat{g}(\infty)=\infty$. By what we showed in Case II, there exists a number $k$ such that if $t_{0} \in(-\infty, \infty), A>0, B>0$, and $\widehat{y}(t)$ is defined for $t_{0} \leq t<\infty$ and satisfies

$$
\widehat{y}^{\prime \prime}(t)+c \hat{y}^{\prime}(t)+\widehat{g}(\widehat{y}(t))=-p(t)
$$

and if $\left|\widehat{y}\left(t_{0}\right)\right| \leq A,\left|\hat{y}^{\prime}\left(t_{0}\right)\right| \leq B$, then $t \geq t_{0} \Rightarrow\left|\hat{y}^{\prime}\left(t_{0}\right)\right| \leq b(A, B)$, and $t \geq t_{0}+T(A, B) \Rightarrow\left|\hat{y}^{\prime}(t)\right| \leq k$.

If $y$ is defined on $\left[t_{0}, \infty\right)$ and satisfies $y^{\prime \prime}+c y^{\prime}+g(y)=p(t)$, then $\hat{y}(t) \equiv$ $-y(t)$ will satisfy (2.18). Hence, if $\left|y\left(t_{0}\right)\right| \leq A$ and $\left|y^{\prime}\left(t_{0}\right)\right| \leq B$, then (2.6) holds for $t \geq t_{0}+T(A, B)$, and (2.7) holds for $t \geq t_{0}$.

Case IV. Suppose that

$$
-\infty=g(-\infty)<g(\xi)<g(\infty)=\infty, \quad \xi \in(-\infty, \infty) .
$$

In this case we can prove Theorem 2.1 by using a Liapunov function (see, e.g. [10 or 12$])$.

If $G(\xi)$ is defined by $G^{\prime}(\xi)=g(\xi)$, and $G(0)=0$, then, by L'Hospital's rule,

$$
G(\xi) /|\xi| \rightarrow \infty \text { as }|\xi| \rightarrow \infty .
$$

Let $\vec{F}(y, v, t)$ be the time dependent vector field given by

$$
\vec{F}(y, v, t)=\operatorname{col}(v, p(t)-c v-g(y)),
$$

which corresponds to the first order system equivalent to the second order differential equation (2.3).

If for $v \geq 2 c_{1} / c$ and $-\infty<y<\infty$, we set

$$
E_{1}(y, v)=v^{2} / 2+G(y)+2 c_{1} y / 3,
$$

then for $(y, v, t) \in(-\infty, \infty) \times\left[2 c_{1} / c, \infty\right) \times(-\infty, \infty)$

$$
\begin{gathered}
\operatorname{grad} E_{1}(y, v) \cdot \vec{F}(y, v, t)=-v\left[c v-p(t)-2 c_{1} / 3\right] \\
\quad \leq-\left(2 c_{1} / c\right)\left[2 c_{1}-c_{1}-2 c_{1} / 3\right]=-2 c_{1}^{2} / 3 c<0 .
\end{gathered}
$$

Similarly, if for $v \leq-2 c_{1} / c$ and $-\infty<y<\infty$, we set

$$
E_{2}(y, v)=v^{2} / 2+G(y)-2 c_{1} y / 3,
$$


then for $(y, v, t) \in(-\infty, \infty) \times\left(-\infty,-2 c_{1} / c\right] \times(-\infty, \infty)$

$$
\begin{gathered}
\operatorname{grad} E_{2}(y, v) \cdot \vec{F}(y, v, t)=v\left[-c v+p(t)-2 c_{1} / 3\right] \\
\leq-\left(2 c_{1} / c\right)\left[2 c_{1}-c_{1}-2 c_{1} / 3\right]=-2 c_{1}^{2} / 3 c .
\end{gathered}
$$

If $-2 c_{1} / c \leq v \leq 2 c_{1} / c$ and $-\infty<y<\infty$, we let

$$
E_{3}(y, v)=v^{2} / 2+G(y)+\frac{c}{3} y v .
$$

Since $(2.8)^{4}$ implies that $(\operatorname{sgn} y) g(y) \rightarrow \infty$ as $|y| \rightarrow \infty$, we may choose $\bar{a}>0$ so large that if $|y| \geq \bar{a}$, then $-c y g(y) / 3+c_{1} c|y|+2 c_{1}^{2} / c \leq-1$. It follows that if $|v| \leq 2 c_{1} / c,|y| \geq a$, and $t \in(-\infty, \infty)$, then

$$
\begin{aligned}
\operatorname{grad} E_{3}(y, v) \cdot \vec{F}(y, v, t) & =v p(t)-2 c v^{2} / 3+c y(p(t)-c v-g(y)) / 3 \\
& \leq-c y g(y) / 3+c_{1} c|y|+2 c_{1}^{2} / c \leq-1 .
\end{aligned}
$$

If $E(y, v)$ is the continuous function defined by

$$
E(y, v)= \begin{cases}E_{1}(y, v) & \text { if } v \geq 2 c_{1} / c, y \in R \\ E_{2}(y, v) & \text { if } v \leq-2 c_{1} / c, y \in R \\ E_{3}(y, v) & \text { if }|v|<2 c_{1} / c, y \in R\end{cases}
$$

Then, from (2.19) we see that

$$
E(y, v) \rightarrow \infty \text { as } y^{2}+v^{2} \rightarrow \infty
$$

We define $E^{\prime}: R^{3} \rightarrow R$ by setting

$$
E^{\prime}\left(y_{0}, v_{0}, t_{0}\right)=\limsup _{s \rightarrow 0^{+}} \frac{1}{s}\left[E\left(y\left(t_{0}+s\right), y^{\prime}\left(t_{0}+s\right)-E\left(y_{0}, v_{0}\right)\right)\right]
$$

where the limsup as $s \rightarrow 0^{+}$is taken over all solutions of (2.3) such that $y\left(t_{0}\right)=y_{0}$ and $y^{\prime}\left(t_{0}\right)=v_{0}$. According to (2.21), (2.23), and (2.25) if $K_{0}$ denotes the set of points $(y, v)$ in $R^{2}$ such that $|y| \leq \bar{a}$ and $|v| \leq 2 c_{1} / c$, then

$$
(y, v) \notin K_{0} \Rightarrow E^{\prime}(y, v, t) \leq-\delta<0
$$

for arbitrary $t \in R$, where $\delta=\min \left\{1,-2 c_{1}^{2} / 3 c\right\}$. Let $A>0, B>0$, and let $y(t)$ be a solution of (2.3) with $\left|y\left(t_{0}\right)\right| \leq A$ and $\left|y^{\prime}\left(t_{0}\right)\right| \leq B$. Let $K_{1}$ be the rectangle consisting of points $(y, v)$ such that $|y| \leq A_{1} \equiv \max \{\bar{a}, A\}$ and $|v| \leq$ $B_{1} \equiv \max \left\{2 c_{1} / c, B\right\}$. By (2.19), there exist numbers $a(A, B)$ and $b(A, B)$ such that if $K_{2}$ denotes the rectangle consisting of points $(y, v)$ such that $|y| \leq a(A, B)$ and $|v| \leq b(A, B)$, then the minimum of $E$ on the boundary of $K_{2}$ is greater than the maximum of $E$ on $K_{1}$. By standard theory of Liapunov functions (see [9, pp. 371-378; 12, Chapter II]) it follows from (2.27) that if $y(t)$ is a solution of (2.3) such that $\left(y\left(t_{0}\right), y^{\prime}\left(t_{0}\right)\right) \in K_{1}$, then $\left(y(t), y^{\prime}(t)\right) \in$ Interior $K_{2}$ for $t \geq t_{0}$. Therefore, if $\left|y\left(t_{0}\right)\right| \leq A$ and $\left|y^{\prime}\left(t_{0}\right)\right| \leq B$, then $|y(t)| \leq$ $a(A, B),\left|y^{\prime}(t)\right| \leq b(A, B)$ for $t \geq t_{0}$, and this gives (2.7).

The same reasoning shows that if $k_{1}$ and $k$ are so large that the minimum of $E(y, v)$ on the boundary of the rectangle $K_{0}^{\prime}=\left\{(y, v)|| y\left|\leq k_{1},\right| v \mid \leq k\right\}$ 
is bigger than the maximum of $E(y, v)$ on $K_{0}$, then if $y(t)$ is a solution of (2.3) with $\left(y\left(t_{0}\right), y^{\prime}\left(t_{0}\right)\right) \in K_{0}$, it follows that $\left(y(t), y^{\prime}(t)\right) \in K_{0}^{\prime}$ for $t \geq t_{0}$.

Let $A>0, B>0$, and let $K_{1}$ and $K_{2}$ be defined as above. If $y(t)$ is a solution of (2.3) with $\left(y\left(t_{0}\right), y^{\prime}\left(t_{0}\right)\right) \in K_{1}$, then $\left(y(t), y^{\prime}(t)\right) \in K_{2}$ for $t \geq t_{0}$. It follows from (2.27) that if

$$
T(A, B) \equiv\left(\max _{K_{2}} E-\min _{K_{2}} E\right) / \delta,
$$

then there exists a number $t_{*} \in\left[t_{0}, t_{0}+T(A, B)\right]$ such that $\left(y\left(t_{*}\right), y^{\prime}\left(t_{*}\right)\right) \in$ $K_{0}$. Since this implies that $\left(y(t), y^{\prime}(t)\right) \in K_{0}^{\prime}$, we see that if $\left|y\left(t_{0}\right)\right| \leq A$ and $\left|y^{\prime}\left(t_{0}\right)\right| \leq B$, then $|y(t)| \leq k_{1}$ and $\left|y^{\prime}(t)\right| \leq k$ if $t \geq t_{0}+T(A, B)$, which gives (2.6).

Up to this point we have assumed that a solution of the initial value problem (2.4) is defined on $\left[t_{0}, \infty\right)$. If in the above arguments we replace this assumption with the assumption that $y(t)$ is defined on $\left[t_{0}, \alpha\right)$, where $\alpha<\infty$, then the same reasoning shows that given $A>0$ and $B>0$, there exists $b(A, B)$ such that if $\left|y\left(t_{0}\right)\right| \leq A$ and $\left|y^{\prime}\left(t_{0}\right)\right| \leq B$, then $\left|y^{\prime}(t)\right| \leq b(A, B)$ for $t_{0} \leq t<\alpha$. This implies that $|y(t)| \leq A+\left(t-t_{0}\right) b(A, B)$ for $t_{0} \leq t<\alpha$. It follows from standard theory that this implies that $y(t)$ is defined on $\left[t_{0}, \infty\right)$, and the proof of Theorem 2.1 is complete.

\section{THE MAIN THEOREM}

In this section we prove

Theorem 3.1. Let $p(t)$ be defined and continuous for $-\infty<t<\infty$. Assume that there exists $c_{1}>0$ such that $|p(t)| \leq c_{1}$ for all $t \in R$ and that there exists a number $p_{0}$ such that for any $a \in R$

$$
\lim _{T \rightarrow \infty} \frac{1}{T} \int_{a}^{a+T} p(t) d t=p_{0}
$$

where the limit exists uniformly with respect to $a \in R$. Suppose that $g: R \rightarrow R$ is continuous and (2.1) holds. Let $c>0$. If $t_{0} \in(-\infty, \infty)$, then in order that there exist a solution of (2.3) which is bounded on $\left[t_{0}, \infty\right)$ it is necessary that

$$
g(-\infty) \leq p_{0} \leq g(+\infty)
$$

and sufficient that

$$
g(-\infty)<p_{0}<g(+\infty)
$$

If

$$
\xi \in(-\infty, \infty) \Rightarrow g(-\infty)<g(\xi)<g(\infty)
$$

then inequalities (3.3) are both necessary and sufficient for the existence of a solution which is bounded on $\left[t_{0}, \infty\right)$. If inequalities (3.3) hold, then given any $A>0$ and $B>0$, there exist numbers $a(A, B)$ and $b(A, B)$ such that if 
$t_{0} \in(-\infty, \infty)$ and $y(t)$ is a solution of (2.3) with $\left|y\left(t_{0}\right)\right| \leq A$ and $\left|y^{\prime}\left(t_{0}\right)\right| \leq B$, then

$$
|y(t)| \leq a(A, B) \quad \text { and } \quad\left|y^{\prime}(t)\right| \leq b(A, B), \quad t \geq t_{0} .
$$

Moreover, there exist fixed numbers $k_{1}$ and $k$ such that given $A>0$ and $B>0$, there exists $T_{0}(A, B)$ such that if $\left|y\left(t_{0}\right)\right| \leq A$ and $\left|y^{\prime}\left(t_{0}\right)\right| \leq B$, then

$$
t \geq t_{0}+T_{0}(A, B) \Rightarrow|y(t)| \leq k_{1} \text { and }\left|y^{\prime}(t)\right| \leq k .
$$

Proof. We first prove the necessity of conditions (3.2) and (3.3) under the assumptions (2.1) and (3.4), respectively.

Assume that conditions (2.1) hold and that $y(t)$ is a solution of $(2.3)$ which is bounded on $\left[t_{0}, \infty\right)$. As we proved in the previous section, $y^{\prime}(t)$ is also bounded on $\left[t_{0}, \infty\right)$. Therefore,

$$
\begin{aligned}
\lim _{T \rightarrow \infty} & \frac{1}{T} \int_{t_{0}}^{t_{0}+T}\left(y^{\prime \prime}(t)+c y^{\prime}(t)\right) d t \\
\quad= & \lim _{T \rightarrow \infty} \frac{1}{T}\left[y^{\prime}\left(t_{0}+T\right)-y^{\prime}\left(t_{0}\right)+c\left(y\left(t_{0}+T\right)-y\left(t_{0}\right)\right)\right]=0 .
\end{aligned}
$$

It follows from (2.3) that

$$
\lim _{T \rightarrow \infty} \frac{1}{T} \int_{t_{0}}^{t_{0}+T}[p(t)-g(y(t))] d t=0
$$

and therefore,

$$
P_{0}=\lim _{T \rightarrow \infty} \frac{1}{T} \int_{t_{0}}^{t_{0}+T} g(y(t)) d t .
$$

Since conditions (2.1) imply that for all $T>0$

$$
g(-\infty) \leq \frac{1}{T} \int_{t_{0}}^{t_{0}+T} g(y(t)) d t \leq g(\infty)
$$

we obtain (3.2).

Suppose now that the stronger condition (3.3) holds. Again, let $y(t)$ be a solution bounded on $\left[t_{0}, \infty\right)$, and let $r>0$ be chosen so that $|y(t)| \leq r$ for all $t \geq t_{0}$. If $d_{*}$ and $d^{*}$ denote the minimum and maximum of $g(\xi)$ for $-r \leq \xi \leq r$ respectively, then from (2.4) we have $g(-\infty)<d_{*} \leq g(y(t)) \leq$ $d^{*}<g(\infty)$ for all $t \geq t_{0}$. The same argument given above shows that (3.7) holds. Therefore, since for all $T>0$ we have

$$
d_{*} \leq \frac{1}{T} \int_{t_{0}}^{t_{0}+T} g(y(t)) d t \leq d^{*}
$$

it follows that $d_{*} \leq p_{0} \leq d^{*}$, and this gives inequalities (3.3); and thus the necessary part of the theorem is established.

In order to prove the sufficiency part of the theorem, we use the following: 
Lemma 3.2. Assume that inequalities (3.3) hold, and let $r_{1}>0$ and $\delta>0$ be chosen so that

$$
\xi \geq r_{1} \Rightarrow g(\xi) \geq p_{0}+3 \delta
$$

and

$$
\xi \leq-r_{1} \Rightarrow g(\xi) \leq p_{0}-3 \delta .
$$

Let $L_{1}>0$ be chosen so that if $a \in(-\infty, \infty)$ and $T \geq L_{1}$, then

$$
p_{0}-\delta \leq \frac{1}{T} \int_{a}^{a+T} p(t) d t \leq p_{0}+\delta .
$$

If $y(t)$ is a solution of (2.3) defined for $t_{1} \leq t \leq t_{2}$ such that $|y(t)| \geq r_{1}$ and $\left|y^{\prime}(t)\right| \leq b$ for $t_{1} \leq t \leq t_{2}$, then

$$
t_{2}-t_{1} \leq \max \left\{L_{1},(c / \delta)\left(\left|y\left(t_{1}\right)\right|-r_{1}\right), 2 b / \delta\right\} .
$$

Proof. We prove the lemma for the case $y(t) \geq r_{1}$ for $t_{1} \leq t \leq t_{2}$; the proof of the case $y(t) \leq-r_{1}$ for $t_{1} \leq t \leq t_{2}$ will then be clear.

Suppose then that $y(t) \geq r_{1}$ and $\left|y^{\prime}(t)\right| \leq b$ for $t_{1} \leq t \leq t_{2}$ and, contrary to the assertion of the lemma,

$$
t_{2}-t_{1}>\max \left\{L_{1},(c / \delta)\left(y\left(t_{1}\right)-r_{1}\right), 2 b / \delta\right\} .
$$

From (3.8) it follows that $g(y(t)) \geq p_{0}+3 \delta$ for $t_{1} \leq t \leq t_{2}$. Therefore, integrating the differential equation (2.3) from $t_{1}$ and $t_{2}$, and using (3.8), we obtain

$$
\begin{aligned}
c\left(y\left(t_{2}\right)-y\left(t_{1}\right)\right) & =y^{\prime}\left(t_{1}\right)-y^{\prime}\left(t_{2}\right)+\int_{t_{1}}^{t_{2}} p(t) d t-\int_{t_{1}}^{t_{2}} g(y(t)) d t \\
& \leq 2 b+\left(t_{2}-t_{1}\right)\left(p_{0}+\delta\right)-\left(t_{2}-t_{1}\right)\left(p_{0}+3 \delta\right) .
\end{aligned}
$$

Since $2 b<\left(t_{2}-t_{1}\right) \delta$, it follows from (3.11) that

$$
y\left(t_{2}\right)<y\left(t_{1}\right)-\left(t_{2}-t_{1}\right) \delta / c<r_{1},
$$

which is a contradiction. This completes the proof of the lemma.

Lemma 3.3. Assume that conditions (3.3) hold, and let $r_{1}, \delta$, and $L_{1}$ be as in the statement of Lemma 3.2. If $y(t)$ is a solution of (2.3) such that $y(t)$ is defined for $\bar{t} \leq t<\infty,|y(\bar{t})| \leq r_{1}$, and $\left|y^{\prime}(t)\right| \leq b$ for $t \geq \bar{t}$, then

$$
|y(t)| \leq r_{1}+(b / 2) \max \left\{L_{1}, 2 b / \delta\right\}, \quad t \geq \bar{t} .
$$

Proof. If $\left[t_{1}, t_{2}\right]$ is a closed and bounded subinterval of $[\bar{t}, \infty)$ such that $\left|y\left(t_{1}\right)\right|=\left|y\left(t_{2}\right)\right|=r_{1}$, and $|y(t)| \geq r_{1}$ for $t_{1} \leq t \leq t_{2}$, then according to Lemma 3.2 we have $t_{2}-t_{1} \leq \max \left\{L_{1}, 2 b / \delta\right\}$. Let us consider the case where $y\left(t_{1}\right)=y\left(t_{2}\right)=r_{1}$, and $y(t) \geq r_{1}$ for $t_{1} \leq t \leq t_{2}$. Let $\xi$ be a number in the interval $\left[t_{1}, t_{2}\right]$ such that $y(t)$ assumes its maximum on the interval $\left[t_{1}, t_{2}\right]$ at $\xi$. Since $\left|y^{\prime}(t)\right| \leq b$ for $t \in\left[t_{1}, t_{2}\right]$, it follows from the mean value theorem that 
$\left|y(\xi)-r_{1}\right|=\left|y(\xi)-y\left(t_{1}\right)\right| \leq b\left(\xi-t_{1}\right)$ and $\left|y(\xi)-r_{1}\right|=\left|y\left(t_{2}\right)-y(\xi)\right| \leq b\left(t_{2}-\xi\right)$. Since either $\xi-t_{1} \leq\left(t_{2}-t_{1}\right) / 2$ or $t_{2}-\xi \leq\left(t_{2}-t_{1}\right) / 2$, it follows that

$$
|y(\xi)| \leq r_{1}+b\left(t_{2}-t_{1}\right) / 2 \leq r_{1}+(b / 2) \max \left\{L_{1}, 2 b / \delta\right\} .
$$

A similar argument shows that if $y\left(t_{1}\right)=y\left(t_{2}\right)=-r_{1}, y(t) \leq-r_{1}$ for $t_{1} \leq t \leq$ $t_{2}$, and $y(t)$ assumes its minimum at $\xi, \xi \in\left[t_{1}, t_{2}\right]$, then

$$
|y(\xi)| \leq r_{1}+(b / 2) \max \left\{L_{1}, 2 b / \delta\right\} .
$$

This proves the lemma.

The sufficiency part of Theorem 3.1 now follows from the last lemma. For, if $t_{0} \in(-\infty, \infty)$ and $y(t)$ is a solution of the initial value problem (2.4), then, according to Theorem 2.1, there exists a number $b, b>0$, depending on $y_{0}$ and $v_{0}$, such that $\left|y^{\prime}(t)\right| \leq b$ for $t \geq t_{0}$. If (3.3) holds and $\left|y_{0}\right| \leq r_{1}$, then

$$
|y(t)| \leq r_{1}+(b / 2) \max \left\{L_{1}, 2 b / \delta\right\}
$$

for all $t, t \geq t_{0}$, and we have established the existence of a solution of (2.3) bounded on $\left[t_{0}, \infty\right)$.

It remains to establish (3.5) and (3.6), assuming (3.3). We assume that these conditions hold throughout the remainder of this section. Suppose that $y(t)$ is a solution of (2.3) with $\left|y\left(t_{0}\right)\right| \leq A$ and $\left|y^{\prime}\left(t_{0}\right)\right| \leq B$, where $A>0, B>0$ are arbitrary. According to Theorem 2.1 there exists $b(A, B)$ such that $\left|y^{\prime}(t)\right| \leq$ $b(A, B)$ for all $t \geq t_{0}$. We consider two cases.

Case I. $A \leq r_{1}$. In this case, by Lemma 3.3,

$$
|y(t)| \leq r_{1}+\frac{b(A, B)}{2} \max \left\{L_{1}, \frac{2 b(A, B)}{\delta}\right\}, \quad t \geq t_{0},
$$

and (3.5) holds. By Theorem 2.1, there exists a number $k$, independent of $A$ and $B$, and a number $T(A, B)$ such that $\left|y^{\prime}(t)\right| \leq k$ for all $t \geq t_{0}+$ $T(a, b)$. Referring to the proof of Lemma 3.3, we see that if $\left[t_{1}, t_{2}\right]$ is a closed subinterval of $\left[t_{0}, \infty\right)$ such that $|y(t)| \geq r_{1}$ for $t \in\left[t_{1}, t_{2}\right]$, then

$$
t_{2}-t_{1} \leq \max \left\{L_{1}, 2 b(A, B) / \delta\right\} \text {. }
$$

Therefore, there exists a number $\bar{t}$ such that $|y(\bar{t})| \leq r_{1}$ and

$$
t_{0}+T(A, B) \leq \bar{t} \leq t_{0}+T(A, B)+\max \left\{L_{1}, 2 b(A, B) / \delta\right\} .
$$

Since $\left|y^{\prime}(t)\right| \leq k$ for $\bar{t} \leq t<\infty$, it follows from Lemma 3.3 that

$$
|y(t)| \leq r_{1}+(k / 2) \max \left\{L_{1}, 2 k / \delta\right\}=k_{1}, \quad t \geq \bar{t} .
$$

Therefore, if we set

$$
T_{0}(A, B)=T(A, B)+\max \left\{L_{1}, 2 b(A, B) / \delta\right\},
$$

then (3.6) holds with $k_{1}$ defined as above. 
Case II. $r_{1}<A$. Assume that $r_{1}<\left|y\left(t_{0}\right)\right| \leq A$. According to Lemma 3.2, there exists a number $t_{*}$ such that

$$
\begin{aligned}
t_{0} & <t_{*} \leq t_{0}+\max \left\{L_{1}, c\left(\left|y\left(t_{0}\right)\right|-r_{1}\right) / \delta, 2 b(A, B) / \delta\right\} \\
& \leq t_{0}+\max \left\{L_{1}, c\left(A-r_{1}\right) / \delta, 2 b(A, B) / \delta\right\},
\end{aligned}
$$

and $\left|y\left(t_{*}\right)\right| \leq r_{1}$.

A repetition of the argument given in Case I shows that if $k_{1}$ is defined by (3.14), then $|y(t)| \leq k_{1}$ and $\left|y^{\prime}(t)\right| \leq k$ if

$$
t \geq t_{*}+T(A, B)+\max \left\{L_{1}, 2 b(A, B) / \delta\right\} .
$$

Therefore, if we set

$$
\begin{aligned}
T_{0}(A, B)= & T(A, B)+\max \left\{L_{1}, 2 b(A, B) / \delta\right\} \\
& +\max \left\{L_{1}, c\left(A-r_{1}\right) / \delta, 2 b(A, B) / \delta\right\}
\end{aligned}
$$

then (3.6) holds. If $\left|y\left(t_{0}\right)\right| \leq r_{1}<A$, then, as shown in Case I, $|y(t)| \leq k_{1}$ and $\left|y^{\prime}(t)\right| \leq k$ for

$$
t \geq t_{0}+T(A, B)+\max \left\{L_{1}, 2 b(A, B) / \delta\right\},
$$

and hence (3.6) will obviously hold with the preceding definition of $T_{0}(A, B)$.

If $r_{1}<y\left(t_{0}\right) \leq A$ and $t$ is defined as above, then since $\left|y^{\prime}(t)\right| \leq b(A, B)$ for $t \geq t_{0}$, it follows from (3.15) that

$$
\begin{aligned}
|y(t)| & \leq\left|y\left(t_{0}\right)\right|+\left(t_{*}-t_{0}\right) b(A, B) \\
& \leq A+b(A, B) \max \left\{L_{1}, c\left(A-r_{1}\right) / \delta, 2 b(A, B) / \delta\right\}
\end{aligned}
$$

for $t_{0} \leq t \leq t_{*}$. It follows from Lemma 3.3 that (3.13) holds for $t \geq t^{*}$. Therefore, if we set

$$
a(A, B)=A+b(A, B) \max \left\{L_{1}, c\left(A-r_{1}\right) / \delta, 2 b(A, B) / \delta\right\},
$$

then (3.5) holds for $t \geq t_{0}$.

If $\left|y\left(t_{0}\right)\right| \leq r_{1}<A$, then, since the right-hand side of inequality (3.13) is less than $a(A, B),(3.5)$ will still hold for $t \geq t_{0}$. This completes the proof of Theorem 3.1.

Remark. By examining the proofs in this section, one notes that if in Theorem 3.1 the assumption

$$
\lim _{T \rightarrow \infty} \frac{1}{T} \int_{a}^{a+T} p(t) d t=p_{0}
$$

exists for all $a$ uniformly in $a$ is replaced by the assumption that

$$
\lim _{T \rightarrow \infty} \frac{1}{T} \int_{t_{0}}^{t_{0}+T} p(t) d t
$$

exists, then if (2.1) holds, (3.2) is necessary for the existence of a solution bounded on $\left[t_{0}, \infty\right)$; while if $(2.1)$ is replaced by (3.4), and (3.16) holds, then 
(3.2) is both necessary and sufficient that all solutions be bounded on $\left[t_{0}, \infty\right)$. However, (3.1) is needed in the next section.

\section{EXISTENCE of A SOlution Bounded on $(-\infty, \infty)$}

Theorem 4.1. Assume that $p$ and $g$ are continuous and that (2.1), (2.2) and (3.1) hold. If (3.3) holds, then there exists a solution $y(t)$ of (2.3) such that $y(t)$ and $y^{\prime}(t)$ are both bounded on $(-\infty, \infty)$.

Proof. For brevity we set $a_{1}=a(1,1), b_{1}=b(1,1)$ in (3.5). For each integer $n, n=1,2, \ldots$, let $y_{n}(t)$ be the solution of the initial value problem

$$
\left\{\begin{array}{l}
y^{\prime \prime}(t)+c y^{\prime}(t)+g(y(t))=p(t), \\
y(-n)=0, \quad y^{\prime}(-n)=0 .
\end{array}\right.
$$

According to (3.5), we have

$$
\left|y_{n}(t)\right| \leq a, \quad\left|y_{n}^{\prime}(t)\right| \leq b \quad \text { for } t \geq-n .
$$

For convenience we define a $C^{1}$-function $z_{n}(t)$ on $(-\infty, \infty)$ for $n=$ $1,2, \ldots$ by setting

$$
z_{n}(t)=\left\{\begin{array}{l}
y_{n}(t), \quad-n \leq t, \\
0, \quad t<-n .
\end{array}\right.
$$

From (4.1), (4.2) and the fact that $|p(t)| \leq c_{1}$ for $t \in(-\infty, \infty)$ we see that the sequences $\left\{z_{n}(t)\right\}_{1}^{\infty}$ and $\left\{z_{n}^{\prime}(t)\right\}_{1}^{\infty}$ are uniformly bounded on $(-\infty, \infty)$, and for each $n, n=1,2, \ldots, z_{n}$ has a piecewise continuous second derivative, which is bounded on $(-\infty, \infty)$ independently of $n$. It follows from Ascoli's lemma and induction that for each integer $m, m=1,2, \ldots$, there exists a sequence of functions $\left\{z_{m, n}(t)\right\}_{n=1}^{\infty}$ such that $\left\{z_{1, n}(t)\right\}_{n=1}^{\infty}$ is a subsequence of $\left\{z_{n}(t)\right\}_{1}^{\infty},\left\{z_{m, n}(t)\right\}_{n=1}^{\infty}$ is a subsequence of $\left\{z_{m-1, n}(t)\right\}_{n=1}^{\infty}$ for $m>1$, and for $m \geq 1$ both of the sequences $\left\{z_{m, n}(t)\right\}_{n=1}^{\infty}$ and $\left\{z_{m, n}^{\prime}(t)\right\}_{n=1}^{\infty}$ converge uniformly with respect to $t$ on the interval $-m \leq t \leq m$. If we denote the $k$ th member of the diagonal sequence $\left\{z_{n, n}(t)\right\}_{1}^{\infty}$ by $w_{k}(t), k=1,2, \ldots$, then for any $t_{1}$ and $t_{2}$, with $-\infty<t_{1} \leq t_{2}<\infty$, both of the sequences $\left\{w_{k}(t)\right\}_{1}^{\infty}$ and $\left\{w_{k}^{\prime}(t)\right\}_{1}^{\infty}$ converge uniformly with respect to $t$ on $\left[t_{1}, t_{2}\right]$. We note that if $t_{1} \in(-\infty, \infty)$ and $-k<t_{1}$, then $w_{k}$ is of class $C^{2}$ on the interval $\left[t_{1}, \infty\right)$, and

$$
w_{k}^{\prime \prime}(t)+c w_{k}^{\prime}(t)+g\left(w_{k}(t)\right)=p(t)
$$

on this interval. Thus, it follows that the sequence $\left\{w_{k}^{\prime \prime}(t)\right\}_{1}^{\infty}$ converges uniformly on bounded subintervals of $(-\infty, \infty)$. Therefore, if we define $w(t)=$ $\lim _{k \rightarrow \infty} w_{k}(t)$ for $t \in(-\infty, \infty)$, then $w \in C^{2}(-\infty, \infty)$ and

$$
w^{\prime \prime}(t)+c w^{\prime}(t)+g(w(t))=p(t) .
$$

Since, according to (4.2), we have $|w(t)| \leq a$ and $\left|w^{\prime}(t)\right| \leq b$ for $t \in(-\infty, \infty)$, the proof of the theorem is complete. 


\section{THE EFFECT OF LARGE DAMPING}

In this section we only assume that $g$ is defined and continuous on some interval. As a by-product of some of the estimates used in the previous sections we obtain

Theorem 5.1. Let $g$ be defined and continuous on some interval, and let $p(t)$ satisfy the conditions of Theorem 3.1. Assume that there exists an interior point $\xi_{0}$ of the domain of $g$ such that $g\left(\xi_{0}\right)=p_{0}, g(\xi)>p_{0}$ for $\xi-\xi_{0}$ small and positive, and $g(\xi)<p_{0}$ for $\xi_{0}-\xi$ small and positive. Given $\alpha>0$, there exist numbers $\gamma_{1}>0, \gamma_{2}>0$ and $c^{*}>0$ such that if $c \geq c^{*}, t_{0} \in(-\infty, \infty)$, and $y(t)$ is a solution of

$$
y^{\prime \prime}(t)+c y^{\prime}(t)+g(y(t))=p(t)
$$

satisfying $\left|y\left(t_{0}\right)-\xi_{0}\right|<\gamma_{1}$ and $\left|y^{\prime}\left(t_{0}\right)\right|<\gamma_{2}$, then

$$
\left|y(t)-\xi_{0}\right|<\alpha, \quad\left|y^{\prime}(t)\right|<\alpha
$$

for $t \geq t_{0}$.

Proof. It suffices to prove the theorem under the additional assumptions that $p_{0}=0$ and $\xi_{0}=0$. For, we note that if $p_{1}(t) \equiv p(t)-p_{0}$ and $g_{1}(\xi) \equiv$ $g\left(\xi+\xi_{0}\right)-p_{0}$, then

$$
\lim _{T \rightarrow \infty} \frac{1}{T} \int_{a}^{a+T} p_{1}(t) d t=0
$$

uniformly with respect to $a \in(-\infty, \infty), p_{1}(t)$ is continuous for $-\infty<t<\infty$, $g_{1}(0)=0, g_{1}(\xi)>0$ for $\xi$ small and positive, and $g_{1}(\xi)<0$ for $\xi$ small and negative. Moreover, $y(t)$ is a solution of (5.1) satisfying (5.2) on $t_{0} \leq t<\infty$ if and only if $z(t) \equiv y(t)-\xi_{0}$ is a solution of

$$
z^{\prime \prime}(t)+c z^{\prime}(t)+g_{1}(z(t))=p_{1}(t)
$$

satisfying $|z(t)|<\alpha$ and $\left|z^{\prime}(t)\right|<\alpha$ for $t_{0} \leq t<\infty$.

Suppose then that the conditions of Theorem 5.1 hold and, in addition, $\xi_{0}=$ $p_{0}=0$. Given $\alpha>0$, choose $r_{1}>0$ so small that $2 r_{1} \leq \alpha, g(\xi)>g(0)=0$ for $0<\xi \leq 2 r_{1}$, and $g(\xi)<0$ for $-2 r_{1} \leq \xi<0$. Let $g^{*}(\xi)$ be the function defined on $(-\infty, \infty)$ by

$$
g^{*}(\xi)= \begin{cases}g\left(2 r_{1}\right) & \text { if } \xi \geq 2 r_{1}, \\ g(\xi) & \text { if }|\xi|<2 r_{1}, \\ g\left(-2 r_{1}\right) & \text { if } \xi<-2 r_{1} .\end{cases}
$$

If $\delta>0$ and $L_{1}>0$ are chosen so that $\xi \geq r_{1} \Rightarrow g^{*}(\xi) \geq 3 \delta, \xi \leq-r_{1} \Rightarrow$ $g^{*}(\xi) \leq-3 \delta$, and

$$
T \geq L_{1} \Rightarrow-\delta \leq \frac{1}{T} \int_{a}^{a+T} p(t) d t \leq \delta
$$


for all $a \in(-\infty, \infty)$, then, according to Lemma 3.3, for any solution $y(t)$ of the differential equation

$$
y^{\prime \prime}(t)+c y(t)+g^{*}(y(t))=p(t)
$$

which satisfies the conditions $\left|y\left(t_{0}\right)\right| \leq r_{1}$ and $\left|y^{\prime}(t)\right| \leq b$, we have

$$
|y(t)| \leq r_{1}+(b / 2) \max \left\{L_{1}, 2 b / \delta\right\}
$$

for all $t \geq t_{0}$.

Let $\gamma_{2}>0$ be chosen so small that $\gamma_{2}<\alpha$ and

$$
\left(\gamma_{2} / 2\right) \max \left\{L_{1}, 2 \gamma_{2} / \delta\right\}<r_{1} \text {. }
$$

If $c_{1}$ and $c_{2}$ are constants such that $|p(t)| \leq c_{1}$ for $-\infty<t<\infty,\left|g^{*}(\xi)\right| \leq c_{2}$ for $-\infty<\xi<\infty$, and if we set $c_{3}=c_{1}+c_{2}$, then, referring to the proof of Theorem 2.1 (Case I), we see from (2.9) that if $y(t)$ is a solution of (5.4) defined on $\left[t_{0}, \infty\right)$, then

$$
\left|y^{\prime}(t)\right| \leq \max \left\{\left|y^{\prime}\left(t_{0}\right)\right|, c_{3} / c\right\}
$$

for $t \geq t_{0}$. It follows that if $c^{*}$ is so large that $c_{3} / c^{*}<\gamma_{2}$, if $c \geq c^{*}$, and $y(t)$ is any solution of (5.4) such that $\left|y^{\prime}\left(t_{0}\right)\right|<\gamma_{2}$, then $\left|y^{\prime}(t)\right|<\gamma_{2}$ on $\left[t_{0}, \infty\right)$.

We claim that if $\gamma_{2}$ and $c^{*}$ are as above and we set $\gamma_{1}=r_{1}$, then the assertion of Theorem 5.1 holds. To see this, we note that if $c \geq c^{*}$ and $\widehat{y}(t)$ is a solution of (5.1) with $\left|\widehat{y}\left(t_{0}\right)\right|<\gamma_{1}$ and $\left|\widehat{y}^{\prime}\left(t_{0}\right)\right|<\gamma_{2}$, then $\widehat{y}(t)$ is also a solution of (5.4) for $t>t_{0}$ as long as $|\widehat{y}(t)| \leq 2 r_{1}$. However, as shown above, any solution $y(t)$ of (5.4) with $\left|y\left(t_{0}\right)\right|<\gamma_{1}=r_{1}$ and $\left|y^{\prime}\left(t_{0}\right)\right|<\gamma_{2}$ satisfies $\left|y^{\prime}(t)\right| \leq \gamma_{2}$ for $t>t_{0}$ and, therefore, by (5.5) and (5.6),

$$
|y(t)| \leq r_{1}+\left(\gamma_{2} / 2\right) \max \left\{L_{1}, 2 \gamma_{2} / \delta\right\}<2 r_{1}
$$

for $t>t_{0}$. This shows that $\widehat{y}(t)$ is defined for $t \geq t_{0},|\widehat{y}(t)|<2 r_{1} \leq \alpha$, and $\left|\widehat{y}^{\prime}(t)\right| \leq \gamma_{2}<\alpha$ for $t \geq t_{0}$. This proves the theorem.

Using the same type of argument which was used to prove Theorem 4.1, one can prove

Theorem 5.2. If the hypotheses of Theorem 5.1 hold, then given any $\alpha>0$ there exists a number $c^{*}>0$ such that for $c \geq c^{*}$ there exists a solution $y(t)$ of (5.1) defined on $(-\infty, \infty)$ such that $\left|y(t)-\xi_{0}\right|<\alpha$ and $\left|y^{\prime}(t)\right|<\alpha$ for all $t$ in $(-\infty, \infty)$.

\section{AN EXAMPLE}

We conclude with an example to illustrate the difference in behavior which can occur when the damping constant $c$ increases from small positive values to large positive values. Let $g$ be an odd $C^{1}$ function such that $g^{\prime}(\xi) \geq 0$ for all $\xi \in(-\infty, \infty), g(\xi)=b \xi$ for $-d \leq \xi \leq d$, where $b>0$ and $d>0$, and such that the $\lim _{\xi \rightarrow \pm \infty} g(\xi)=g( \pm \infty)$ are finite. In engineering literature, $g$ 
is referred to as a saturation function since the corresponding restoring force acts as a linear spring for displacements within a certain range, but saturates, i.e. becomes nearly constant, for large displacements.

An elementary analysis shows that all solutions of

$$
y^{\prime \prime}(t)+g(y(t))=0
$$

are periodic and that the period is a nondecreasing function of the amplitude (see [7]). This implies that there exists a nonconstant periodic solution $y_{0}(t)$ of (6.1) of period $L, L>0$, such that all $L$-periodic solutions of the variational equation

$$
z^{\prime \prime}(t)+g^{\prime}\left(y_{0}(t)\right) z(t)=0
$$

are constant multiples of $y_{0}^{\prime}(t)$ (see [7]). The theory developed in [7] shows that if $f(t)$ is a continuous $L$-periodic function such that

$$
\int_{0}^{L} y_{0}^{\prime}(t) f(t) d t=0
$$

and

$$
\int_{0}^{L} y_{0}^{\prime \prime}(t) f(t) d t \neq 0
$$

then for $\varepsilon$ sufficiently small and positive and $c$ sufficiently small and positive (the magnitude depending on $\varepsilon$ ) there exists an $L$-periodic solution of

$$
y^{\prime \prime}(t)+c y^{\prime}(t)+g(y(t))=\varepsilon f(t)
$$

which is close to $y_{0}(t)$ in the $C^{1}$-sense. A particular $f$ that works is $f(t)=$ $y_{0}^{\prime \prime}(t)$. The same result guarantees, for suitably restricted positive $\varepsilon$ and $c$, the existence of an $L$-periodic solution of (6.4) close to $-y_{0}(t)$ in the $C^{1}$-sense. Finally, for $c$ and $\varepsilon$ small and positive, there exists a small amplitude solution of (6.4) which is actually a solution of the linear differential equation

$$
y^{\prime \prime}(t)+c y^{\prime}(t)+b y(t)=\varepsilon f(t) .
$$

Thus, for suitable positive constants $c$ and $\varepsilon,(6.4)$ has at least two $L$-periodic solutions.

In contrast, we now show that if $p(t)$ satisfies the hypotheses of Theorem 3.1 -as any continuous periodic or almost periodic function will-and $g$ is the saturation function described above, then if $\left|p_{0}\right|<b d$ and $c$ is sufficiently large and positive, there exists a unique solution of $(2.3)$ bounded on $(-\infty, \infty)$, which is asymptotically stable and globally attracting.

Suppose then that $\left|p_{0}\right|<b d$ and let $\xi_{0}$ be the unique point in $(-d, d)$ such that $g\left(\xi_{0}\right)=p_{0}$. Let $p_{1}(t)=p(t)-p_{0}$ and $g_{1}(\xi)=g\left(\xi+p \xi_{0}\right)-p_{0}$. Then (5.3) holds, $g_{1}(0)=0$, and $g_{1}$ is linear on an interval centered at 0 . Moreover, there is a one-to-one correspondence between solutions of

$$
z^{\prime \prime}(t)+c z^{\prime}(t)+g_{1}(z(t))=p_{1}(t)
$$


by means of the transformation $y(t)=z(t)-\xi_{0}$.

Let $r_{1}>0$ be so small that $g_{1}(\xi)$ is linear in the range $-2 r_{1} \leq \xi \leq 2 r_{1}$, and let $\delta>0$ be so small that $g_{1}(\xi)>3 \delta$ if $\xi \geq r_{1}$ and $g_{1}(\xi)<-3 \delta$ if $\xi \leq-r_{1}$. Let $L_{1}>0$ be so large that

$$
-\delta<\frac{1}{T} \int_{a}^{a+T} p_{1}(t) d t<\delta
$$

if $a \in(-\infty, \infty)$ and $T \geq L_{1}$.

Let $c_{2}$ be the supremum of $\left|g_{1}(\xi)\right|$ on $(-\infty, \infty)$, and let $c_{3}=c_{1}+c_{2}$. According to (2.10), if $z(t)$ is a solution of (6.5) such that $\left|z\left(t_{0}\right)\right| \leq B$, then there exists $T(B)$ such that if $t \geq t_{0}+T(B)$ then $|z(t)| \leq 2 c_{3} / c \equiv k$.

Let $c^{*}>0$ be so large that $\left(c_{3} / c^{*}\right) \max \left\{L_{1}, 4 c_{3} / c^{*}\right\}<r_{1}$, and suppose that $c>c^{*}$. It follows from (3.14) that, given $A>0$ and $B>0$, if $z(t)$ is a solution of (6.5) such that $\left|z\left(t_{0}\right)\right| \leq A$ and $\left|z^{\prime}\left(t_{0}\right)\right| \leq B$ for some $t_{0}$ in $(-\infty, \infty)$, then there exists $T_{0}(A, B)$ such that for $t \geq t_{0}+T_{0}(A, B)$ we have $\left|z^{\prime}(t)\right| \leq k$ and

$$
|z(t)| \leq r_{1}+(k / 2) \max \left\{L_{1}, 2 k / \delta\right\}<2 r_{1} .
$$

Thus it follows that if $z(t)$ is a solution of $(6.5)$ which is bounded on $(-\infty, \infty)$ and $c>c^{*}$, then $|z(t)|<2 r_{1}$ for all $t$ in $(-\infty, \infty)$. Therefore, since $g_{1}(\xi)=$ $b \xi$ for $|\xi|<2 r_{1}, z(t)$ is a solution of the linear differential equation

$$
z^{\prime \prime}(t)+c z^{\prime}(t)+b z(t)=p_{1}(t) .
$$

Since the homogeneous differential equation

$$
z^{\prime \prime}(t)+c z^{\prime}(t)+b z^{\prime}(t)=0
$$

has no solution bounded on $(-\infty, \infty)$ other than $z \equiv 0$, it follows from standard theory (see, e.g., [4, Chapter IV]) that (6.6) has a unique solution bounded on $(-\infty, \infty)$. Therefore, for $c \geq c^{*}$ it follows that (6.5), and hence (2.3), has a unique solution bounded on $(-\infty, \infty)$.

Let $c \geq c^{*}$, and let $z_{0}(t)$ be the unique solution of (6.5) bounded on $(-\infty, \infty)$. If $A>0, B>0$, and $\widehat{z}(t)$ is another solution of (6.5) such that $\left|\widehat{z}\left(t_{0}\right)\right| \leq A$ and $\left|\widehat{z}^{\prime}\left(t_{0}\right)\right| \leq B$ for some $t_{0} \in(-\infty, \infty)$, then, as shown above, $|\widehat{z}(t)| \leq 2 r_{1}$ for $t \geq t_{0}+T_{0}(A, B)$; thus $z_{0}(t)$ and $\widehat{z}(t)$ are solutions of (6.6) for $t \geq t_{0}+T_{0}(A, B)$. Since the difference of these two solutions is a solution of (6.7), and since the zero solution of (6.7) is globally asymptotically stable, we see that $\widehat{z}(t)-z_{0}(t) \rightarrow 0$ as $t \rightarrow \infty$. This establishes our claim that the unique solution of $(2.3)$, which is bounded on $(-\infty, \infty)$, is globally asymptotically stable.

\section{REFERENCES}

1. J. Bebernes and M. Martelli, Periodic solutions for Liénard systems, Boll. Un. Mat. Ital. A (5) 16 (1979), 398-405.

2. A. S. Besicovitch, Almost periodic functions, Cambridge Univ. Press, 1935. 
3. W. A. Coppel, Stability and asymptotic behavior of differential equations, Heath, 1965.

4. P. O. Frederickson and A. C. Lazer, Necessary and sufficient damping in a second order oscillator, J. Differential Equations 5 (1969), 262-270.

5. J. K. Hale, Ordinary differential equations, Wiley, 1969.

6. A. C. Lazer, On Schauder's fixed point theorem and forced second-order nonlinear oscillations, Math. Anal. Appl. 21 (1968), 421-425.

7. W. S. Loud, Periodic solutions of $x^{\prime \prime}+c x^{\prime}+g(x)=\varepsilon f(t)$, Mem. Amer. Math. Soc. No. 31 (1959).

8. N. Rouche and J. Mawhin, Equations différentielles ordinaires. Stabilité et solutions periodiques, Masson, Paris, 1973 (translated by Pitman, 1980).

9. G. E. H. Reuter, Boundedness theorems for nonlinear differential equations of the second order, J. London Math. Soc. 27 (1952), 48-58.

10. G. Sansone and R. Conti, Nonlinear differential equations, Macmillan, 1964.

11. J. R. Ward, Jr., Periodic solutions of a class of ordinary differential equations, Proc. Amer. Math. Soc. 78 (1980), 350-352.

12. T. Yoshizawa, Stability theory of Liapunov's second direct method, Math. Soc. Japan, 1966.

Department of Mathematics, Computer Science, and Statistics, University of Texas at San Antonio, San Antonio, Texas 78285-0664 